\title{
Preface: Special Issue on WADS 2017
}

๑) Springer Science+Business Media, LLC, part of Springer Nature 2019

This special issue contains selected papers from those presented at the 15th International Algorithms and Data Structures Symposium (WADS 2017), which was held from July 31 to August 2, 2017, in St. John's, Newfoundland, Canada. WADS, which alternates with the Scandinavian Symposium and Workshops on Algorithm Theory, SWAT, is a forum for researchers in the area of design and analysis of algorithms and data structures.

In response to the call for papers, 109 papers were submitted. From these submissions, the Program Committee selected 49 papers for presentation at WADS 2017 , using a combination of online discussion in EasyChair and a one-day video conference. From these 49, the Program Committee selected four outstanding algorithms papers for a special issue in Algorithmica. Extended versions of these papers underwent a rigorous refereeing process. They are presented here:

- Approximating Small Balanced Vertex Separators in Almost Linear Time, by Sebastian Brandt and Roger Wattenhofer

- Modular Circulation and Applications to Traffic Management, by Philip Dasler and David M. Mount

- Faster Algorithm for Truth Discovery via Range Cover, by Ziyun Huang, Hu Ding, and Jinhui Xu

- An EPTAS for Scheduling on Unrelated Machines of Few Different Types, by Klaus Jansen and Marten Maack

We gratefully acknowledge the support of the WADS 2017 sponsors: Memorial University of Newfoundland, The Fields Institute for Research in Mathematical Sciences, Elsevier, and Springer.

July 17,2019

Faith Ellen and

Toronto and Ottawa, Canada Jörg-Rüdiger Sack

Publisher's Note Springer Nature remains neutral with regard to jurisdictional claims in published maps and institutional affiliations. 